\title{
Congenital Plasmodium Vivax Malaria with Maternal TORCHInfection - A Diagnostic Dilemma
}

\author{
Soutrik Seth ${ }^{1}$,Soumita Sadhukhan ${ }^{2}$, Rajib Das ${ }^{3}$, Biswajit Biswas ${ }^{4}$ \\ ${ }^{1,3,4}$ Department Of Pediatrics, Burdwan Medical College And Hospital,Burdwan,WB,India \\ ${ }^{2}$ Department Of Medicine, Command Hospital (Eastern Command),Kolkata,WB,India
}

\begin{abstract}
Congenital malaria is a rare entity but not an uncommon one, particularly in areas of endemicity. Often the diagnosis is complicated by sepsis and many a times its overlooked.Unnecessary investigations and ineffective treatment can be avoided if congenital malaria be distinguished from its close differentials by specific tests. We report here a case of plasmodium vivax positive malaria in a 23 days old neonate born to a primigravida with TORCH infection .
\end{abstract}

Keywords :Congenital malaria ,Endemicity, Neonate , Plasmodium Vivax , TORCH infection

\section{Introduction}

Malaria is a public health concern in our country. India accounts for about $70 \%$ of the total malaria incidence in the South-East Asia Region according to world malaria report 2015.[1]In newborns it is an equally important health issue related to mortality. It can be either acquired antenatally via placenta or postnatally through mosquito bite .Malaria in this age group is very rare because of various defensemechanisms . Congenital malaria is assumed to occur within 10-30 days postnatal life can be delayed even for weeks to months.[2,3]Presentation of neonatal malaria is unlike adults . Common features include irritability ,hepatosplenomegaly, poor feeding and anemia similar to neonatal sepsis . Prognosis is excellent .Breast feeding can be safely continued .Unified efforts are required to control malaria in newborns .

\section{Case Report}

A 23 day old baby girlwas admitted in sick newborn care unit with complaints of excessive cry , irritability, refusal to feed along with abdominal distension and intermittent fever . Baby was born out of nonconsanguineous marriage by normal vaginal delivery to primigravida mother. Antenatal intranatal and postnatal periods were uneventful except for a low grade intermittent feverin the mother 2 weeks prior to delivery. Baby was immunised as per national immunisation schedule .

On physical examination, baby weighed $2.2 \mathrm{kgs}$, measured $50 \mathrm{~cm}$ in length, head circumference measured $33 \mathrm{~cm}$ and abdominal girth was $34 \mathrm{cms}$. Anterior frontanelle was open, measuring $3 \mathrm{~cm}$ by $1 \mathrm{~cm}$. Skin temperature was raised on admission . The baby appeared pale and irritable (Fig.), heart rate was 158/min , respiratory rate was $52 / \mathrm{min}$ with mild subcostal retraction. Abdomen was soft but distended, with liver palpable $5 \mathrm{~cm}$ below right costal margin in midclavicular line, span being $10 \mathrm{cms}$. Spleen was also palpable $8 \mathrm{~cm}$ below left costal margin .There was no significant lymphadenopathy, rash or ascites . Intestinal peristaltic sound was normal .

A initial diagnosis of neonatal sepsis was made and antibiotics were started after drawing blood samples for routine investigations. The following reportscame :Hb - $5 \mathrm{gm} \%$, TLC $-10,200 / \mathrm{cmm}$, N55L41E1M3, platelet count $-1.5 \mathrm{lakh} / \mathrm{cmm}, \mathrm{CRP}-12 \mathrm{mg} / \mathrm{dl}, \mathrm{Na}+136 \mathrm{mEq} / \mathrm{L}, \mathrm{K}+5.2 \mathrm{mEq} / \mathrm{L}$, urine RE normal , total bilirubin $-0.9 \mathrm{mg} / \mathrm{dl}$ ( UC $0.6+\mathrm{C} 0.3$ ), SGPT - 48U/L, SGOT - 40U/L, alkaline phosphatase292U/L, total protein $-6.3 \mathrm{~g} / \mathrm{dl}$, BactAlert- negative , CSF -15 cells N10L90. Accordingly one unit of Packed cell was transfused and even after 2 days there was no improvement. So further investigations were ordered .Hemoglobin electrophoresis - normal, metabolic screening - negative, reticulocyte count $-2.3 \%$, TORCH titre - serum IgGs elevated with IgMs in normal limits ( indicating antenatalmaternal TORCH infections ). Peripheral blood smear showed anisocytosis, poikilocytosis with hypochromia and schizonts of plasmodium vivax ( Fig. 2 ). Peripheral smear was repeated yielding same results and rapid antigen testing by malariakitwas also positive for PlasmodiumVivax infection .(Fig. 3).The history of mother was reevaluated and peripheral blood smear was drawn with Kit testing. Both gametocytes and schizontsof plasmodium vivax were demonstrated in mother also . (Fig. 4). Unfortunately placental histology forparasitemiadetectionwas beyond scope .

Both the mother and baby were started on chloroquine. Once G6PD levels came to be normal in baby, mother was started on primaquine for radical cure and breast feeding continued .The baby started improving with $24 \mathrm{hrs}$ of antimalarial medication and both mother and baby successfully discharged after 5 days .Repeat peripheral smears at followup after 14 days were negative . 


\section{Discussion}

Malaria in pregnancy and newborn accounts for more than 300,000 fetal and infant deaths and 2,500 maternal deathsworldwide annually.[4]Newbornsare normally protected from malarial infections by variety of defense mechanism like maternal antibodies, presence of fetal haemoglobin, higher levels of serum potassium.[3,5]Congenital malaria was first described in 1876.[4,6]Malaria in pregnancy can cause preterm labour, growth restriction , miscarriage and other adverse fetal outcome.[4]

Usually congenital malaria manifest at 10-30 days postnatal life although cases have been found as late as 15 months after birth.[4]It has been postulated that spread occurs possibly through direct penetration of chorionic villi, through premature placental separation and physiological transfusion of maternal blood before cord clamping.[3,7] The most common findings in congenital malaria, as in our case, are irritability , hepatosplenomegaly and refusal to suck mimicking neonatal sepsis. $[3,4,8]$ Unusual presentations like cholestasis may also be present. $[9,10]$ Often the level of parasitemia is low in pregnant women to be detected antenatally, as in our case, and have to be reevaluated later. Often other intrauterine infections may complicate the clinical scenario like TORCH infections as in our case .Thus for any newborn presenting with features of sepsis coming from a malaria endemic region like ours, the clinician should have a level of suspicion to detect congenital or neonatal malaria .

Demonstration of placental parasitemiais gold standard for diagnosing congenital malaria and is possible only when mother is antenatallydiagnosed.[11]Confirmation of diagnosis is normally done by demonstrating the parasite within red corpuscles. Treatment should be initiated as soon as diagnosis is confirmed with schizontocides like chloroquine .For complicated malaria or chloroquine resistant PlasmodiumFalciparummalaria,intravenous quinine or artesunate are used. In congenital malaria, as there are no forms that invade the liver, treatment for exoerythrocytic cycle is not needed.[3,7] The global drive for improvement of perinatal outcome has made congenital malaria a relevant public health issue.

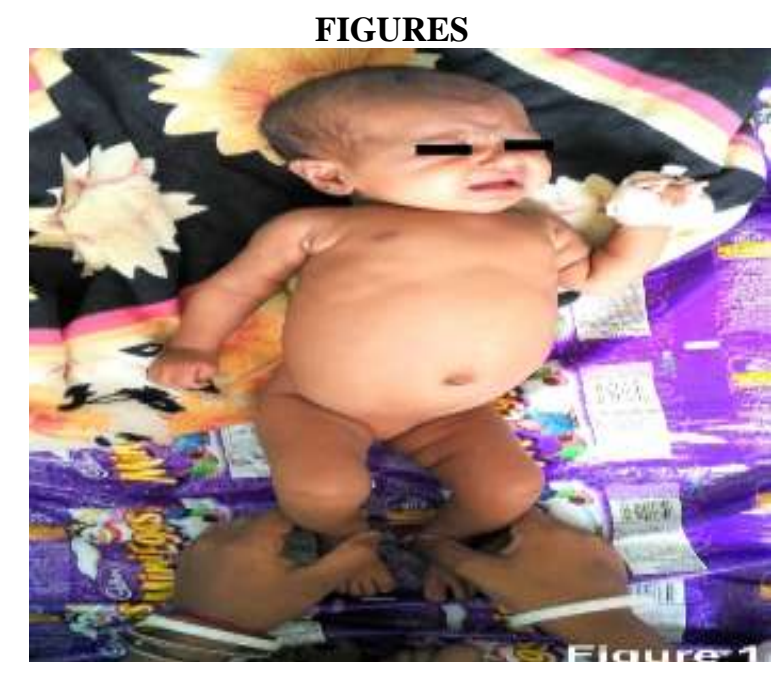

Figure 1 . Baby on admission, irritable and inconsolable cry

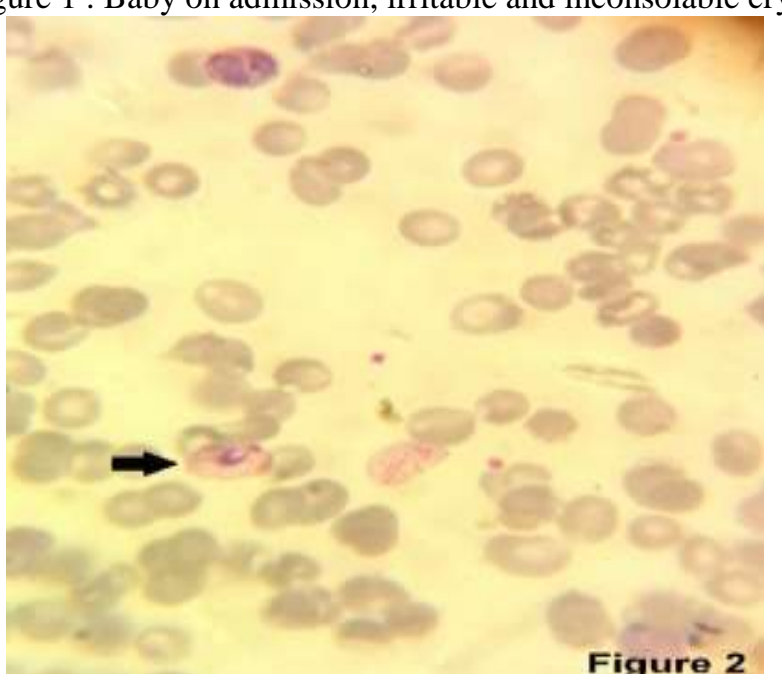


Figure 2. Peripheral blood smear of baby showing schizonts of Plasmodium vivax (arrow), hypochromia and anisocytosis-poikilocytosis

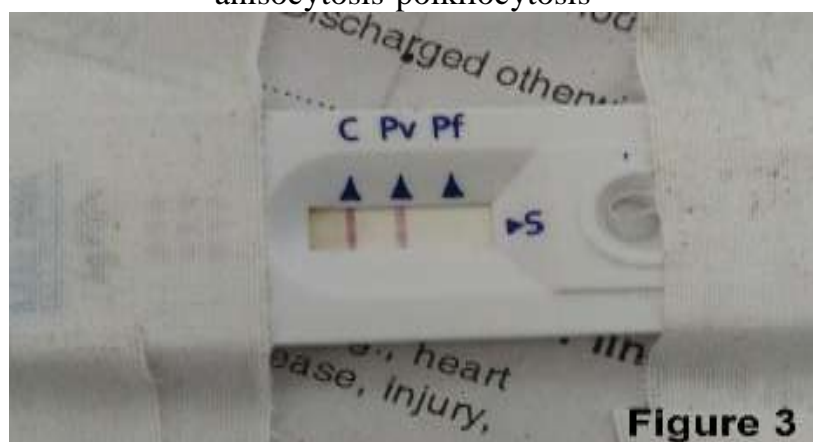

Figure 3 .Malaria Kit test showing Plasmodium Vivax positive in baby

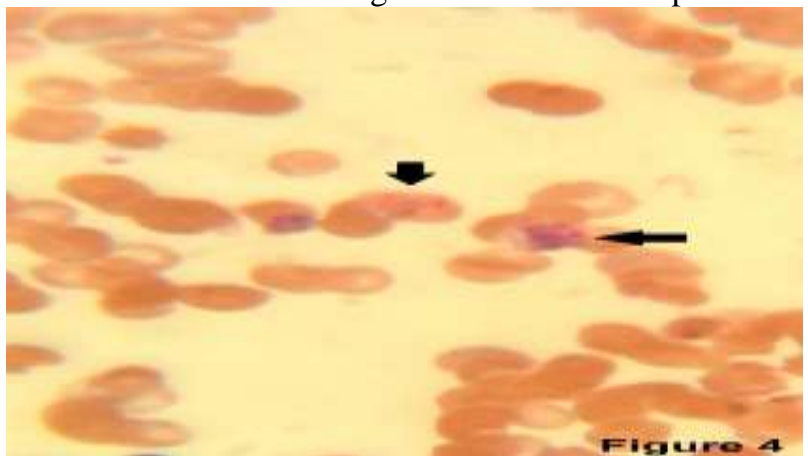

Figure 4 .Peripheral blood smear of mother showing gametocytes and schizonts of Plasmodium vivax(arrows)

\section{Conclusion}

Congenital malaria is an important public health issue related to perinatal mortality . This case suggest the importance of considering malaria as an important differential to neonatal sepsis particularly in endemic countries like India. Often neonatal malaria may have unusual presentations like cholestasis . Treatment with antimalarials is found to be very effective, so early diagnosis and treatment should be done to improve health and reduce hospital stay. Congenital malaria needs to be addressed more promptly .

\section{References}

[1]. Internet link http://www.nvbdcp.gov.in/Doc/National-framework-for-malaria-elimination-in-India-2016\%E2\%80\%932030.pdf, Date accessed: 04 Oct. 2016.

[2]. Ahmad Hashemzadeh ,FarhadHeydarian; Congenital malaria in a neonate; Archives of Iranian Medicine, Volume 8, Number 3, July 2005;226-228

[3]. Uneke CJ:Congenital malaria: an overview. Tanzan J Health Res. 2011, 13: 264-280.

[4]. Rai P, Majumdar K, Sharma S, Chauhan R, Chandra J. Congenital malaria in a neonate: case report with a comprehensive review on differential diagnosis, treatment and prevention in Indian perspective. Journal of Parasitic Diseases: Official Organ of the Indian Society for Parasitology. 2015;39(2):345-348. doi:10.1007/s12639-013-0342-1.

[5]. Gandhi A., Garg K., WadhwaN.. Neonatal Plasmodium vivax malaria: an overlooked entity. The Journal of Infection in Developing Countries, North America, 5, mar. 2011.Available at: <http://www.jidc.org/index.php/journal/article/view/21727650>. Date accessed: 04 Oct. 2016.

[6]. Romand S, Bourée P, Gelez J, Bader-Meunier B, Bisaro F, Dommergues JP. 1994. Congenital malaria. A case observed in twins born to an asymptomatic mother. Journal: Pressemédicale (Paris, France: 1983) (Presse Med) published in France. (Language: fre) 1994 Apr-May;23(17):797-800.

[7]. Sharma P, Jora R, Gupta V ,Chaturvedi A ; Malaria in a 10-day old infant: A case report, Pediatric Infectious disease;5;(2013);6971

[8]. Poespoprodjo JR, Hasanuddin A, Fobia W, et al. Severe Congenital Malaria Acquired in utero. The American Journal of Tropical Medicine and Hygiene. 2010;82(4):563-565. doi:10.4269/ajtmh.2010.09-0744.

[9]. Gathwala G, Dalal P, Gupta M. Congenital malaria with atypical presentation: A series of three case reports. J ClinNeonatol2015;4:206-8

[10]. Sharat V, Abdus S B. Unusual Clinical Presentation of Congenital Malaria: Cholestasis in Newborn. Adv Res GastroenteroHepatol. 2016; 1(5): 555-573. DOI: 10.19080/ARGH.2016.01.555-573

[11]. Rogerson SJ, Mkundika P, KanjalaMK ;Diagnosis of Plasmodium falciparum Malaria at Delivery: Comparison of Blood Film Preparation Methods and of Blood Films with Histology ; J. Clin. Microbiol. April 2003 vol. 41 no. 4 1370-1374; doi: 10.1128/JCM.41.4.1370-1374.2003 